\title{
Transmutation of a part of the extracted material of brass electrodes with pulses of electric current in an aqueous solution of $\mathrm{NaCl}$
}

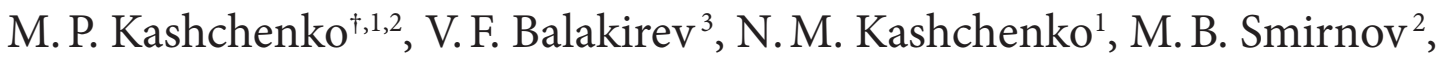 \\ Yu. L. Chepelev², V. V. Ilyushin², N. V. Nikolaeva ${ }^{4}$, V. G. Pushin ${ }^{1,4}$ \\ †mpk46@mail.ru \\ ${ }^{1}$ Ural Federal University n. a. the first President of Russia B. N. Yeltsin, 19 Mira St., Yekaterinburg, 620002, Russia \\ ${ }^{2}$ Ural State Forest Engineering University, 37 Sibirskii Tract, Yekaterinburg, 620100, Russia \\ ${ }^{3}$ The Institute of Metallurgy UB RAS, 101 Amundsen St., Yekaterinburg, 620016, Russia \\ ${ }^{4}$ M. N. Miheev Institute of Metal Physics UB RAS, 18 S. Kovalevskaya St., Yekaterinburg, 620990, Russia
}

In the electroplasma version of the electrolysis of water and aqueous solutions of salts, synthesis of a fairly wide range of chemical elements is observed. In the original installation, the erosion of the electrodes is not significant and the main contribution to the formation of chemical elements precipitated as a solid powder is their synthesis from the substances in the solution. On the contrary, in the simplified installation proposed for demonstrating the synthesis of elements, the erosion of the electrodes is significant. Therefore, it is considered that only elements (from the solid sediment) that are not included in the composition of electrodes are synthesized. To build up the experimental database, systematize and analyze them, we collected a simplified experimental set-up that allows for pulsed electrical discharges in water and aqueous salt solutions using an oscillatory circuit tuned to resonance with a supply voltage $(220 \mathrm{~V}, 50 \mathrm{~Hz})$. Aqueous solution of $\mathrm{NaCl}$ with a concentration of $0.1 \mathrm{~g} / \mathrm{l}$ was used in this experiment. Brass was used as the material of the hollow tubular electrodes. The starting potential difference was $560 \mathrm{~V}$. The gap between the electrodes was $0.7 \mathrm{~mm}$. The electrodes were arranged vertically. Between the upper and lower vessels, the water moved by itself. Each series of discharges was accompanied by the appearance of a portion of a liquid of dark (black) color with subsequent precipitation. The analysis of the sediment showed that along with the products of simple erosion of the electrodes $(\mathrm{Cu}$ and $\mathrm{Zn}$ ), there are synthesized elements $(\mathrm{Mg}, \mathrm{Si}, \mathrm{S}, \mathrm{Al}$, Fe and several others), which confirms the known data. In addition, spherical particles (with diameters of up to $1 \mu \mathrm{m}$ ) have been identified as the main component of which is $\mathrm{Fe}$. The appearance of such particles most likely indicates the possibility of extracting the material from the region of local melting of the electrodes accompanied by copper and zinc transmutations. A discussion of the mechanism of transmutation shows that a significant difference in the isotopic composition of iron from the natural one can be expected.

Keywords: electrolysis, erosion of electrodes, transmutation of extracted material, quasipositronium, synthesis of elements.

УДК: 539.17

\section{Трансмутация части экстрагированного материала латунных электродов при импульсах электрического тока в водном растворе $\mathrm{NaCl}$}

Кащенко М. П. ${ }^{\dagger, 1,2}$, Балакирев В. Ф. ${ }^{3}$, Кащенко Н. М. ${ }^{1}$, Смирнов М. Б. ${ }^{2}$, Чепелев Ю. Л. ${ }^{2}$, Илюшин В. В. ${ }^{2}$, Николаева Н. В. ${ }^{4}$, Пушин В. Г. ${ }^{1,4}$

'Уральский федеральный университет им. первого президента России Б. Н. Ельцина, ул. Мира, 19, Екатеринбург, 620002, Россия

${ }^{2}$ Уральский государственный лесотехнический университет, Сибирский тракт, 37, Екатеринбург, 620100, Россия

${ }^{3}$ Институт металлургии УрО РАН, ул. Амундсена, 101, Екатеринбург, 620016, Россия

${ }^{4}$ Институт физики металлов им. М.Н. Михеева, УрО РАН, ул. С. Ковалевской, 18, Екатеринбург, 620990, Россия 
При электроплазменном варианте электролиза воды и водных растворов солей наблюдается синтез достаточно широкого набора химических элементов. В оригинальной установке эрозия электродов невелика, и основной вклад в образование химических элементов, выпадающих в осадок, дает их синтез из веществ, входящих в состав раствора. Напротив, в упрощенной установке, предложенной для демонстрации синтеза элементов, эрозия электродов велика. Поэтому считается, что из элементов твердого осадка синтезируются только те, что не входят в состав электродов. Для наращивания базы экспериментальных данных, их систематизации и анализа, нами была собрана упрощенная установка, позволяющая осуществлять импульсные электрические разряды в воде и водных растворах солей с использованием колебательного контура, настроенного в резонанс с питающим напряжением (220 В, 50 Гц). В данном эксперименте использовался водный раствор $\mathrm{NaCl}$ с концентрацией 0.1 г/л. В качестве материала полых трубчатых электродов применялась латунь. Стартовая разность потенциалов составляла 560 В. Зазор между электродами составлял 0.7 мм. Электроды располагались вертикально. Между верхним и нижним сосудом вода двигалась самотоком. Каждая серия разрядов сопровождалась появлением порции жидкости с темной (черной) окраской с последующим выпадением осадка. Анализ показал, что, наряду с продуктами простой эрозии электродов ( $\mathrm{Cu}$ и $\mathrm{Zn})$, имеются синтезированные элементы ( $\mathrm{Mg}, \mathrm{Si}, \mathrm{S}, \mathrm{Al}, \mathrm{Fe}$ и ряд других), что подтверждает известные данные. Кроме того, идентифицированы шарообразные частицы (с диаметрами до 1 мкм), основным компонентом состава которых является Fе. Появление таких частиц указывает, скорее всего, на возможность экстрагирования материала из области локального плавления электродов, сопровождающегося трансмутацией меди и цинка. Обсуждение механизма трансмутации показывает, что можно ожидать значительного отличия изотопного состава железа от природного.

Ключевые слова: электролиз, эрозия электродов, трансмутация экстрагированного материала, квазипозитроний, синтез элементов.

\section{1. Введение}

Оригинальная установка плазменного электролиза воды демонстрировала, согласно [1], пример реализации низкоэнергетических ядерных реакций синтеза химических элементов при малой эрозии электродов. Напомним, в [1] синтез связывался с возникновением устойчивого плазменного разряда между электродами, отстоящими на расстояние $(1-1.5) D$, где $D-$ внутренний диаметр полых электродов (для электродов с диаметром до $50 \mathrm{Mм}$ ). Возможное объяснение механизма синтеза требует расширения традиционных представлений $[3,4]$. В то же время целесообразно наращивание базы экспериментальных данных, их систематизации и анализа, как с целью независимого подтверждения известных фактов синтеза элементов, так и установления новых вариантов образования элементов и уточнения условий их формирования. Для решения части задач, согласно [2], была собрана упрощенная, по сравнению c [1], установка, позволяющая осуществлять импульсные электрические разряды в воде и водных растворах солей с использованием колебательного контура, настроенного в резонанс с питающим напряжением (220 В, 50 Гц). В упрощенном варианте отсутствует первый запускающий разряд, внешнее магнитное поле, специальная заточка торцов основных трубчатых электродов. В результате, вместо формирования устойчивого плазмоида имеет место серия разрядов, сопровождающаяся значительной эрозией электродов. В упрощенной установке $D=8$ мм, зазор между медными электродами составлял 0.5-0.7 мм.

В данном эксперименте для улучшения проводимости использовался водный раствор поваренной соли с концентрацией 0.1 г/л (в [2] с той же целью использовалась бура). В качестве материала полых трубчатых электродов применялась латунь (63.77 ат.\% Cu,
36.23 ат.\% Zn). Стартовая разность потенциалов составляла 560 В. Электроды располагались вертикально. Между верхним и нижним сосудом вода двигалась самотоком. Каждая серия разрядов сопровождалась, аналогично [2], появлением порции жидкости с темной (черной) окраской с последующим выпадением осадка.

Цель работы состоит в проведении анализа (по стандартной методике [5]) химического состава фрагментов твердого порошка, получаемого после электролиза, для уточнения выводов исследований, ограничивающихся интегральным химическим анализом $[1,2]$.

\section{2. Химический состав шарообразных частиц, возникших при эрозии катодов}

В [2] предполагалось, что все элементы твердого осадка, не входящие в состав электрода, синтезируются из воды. В частности, значительную долю среди таких элементов составляло железо, что качественно соответствует данным [1]. Выполненный нами микроанализ полученного порошка продемонстрировал неоднородное распределение возникающих элементов внутри фрагментов порошка. Часть фрагментов не имела какой-либо правильной формы и включала несколько локальных областей (зерен) с отличающимся составом. Но наблюдались и шарообразные частицы, диаметры которых могли достигать нескольких микрон.

Так, на Рис. 1 приведен фрагмент, содержащий 4 достаточно крупные частицы, однако видны и существенно меньшие. Близкие к шарообразной формы частиц указывают, по-видимому, на их экстракцию из материала катодов, поверхностные слои которых испытывают локальное плавление во время электрических разрядов. В связи с этим ожидаемым, согласно [2], был бы химический состав частиц, совпадающий с составом электродов. Действительно, 


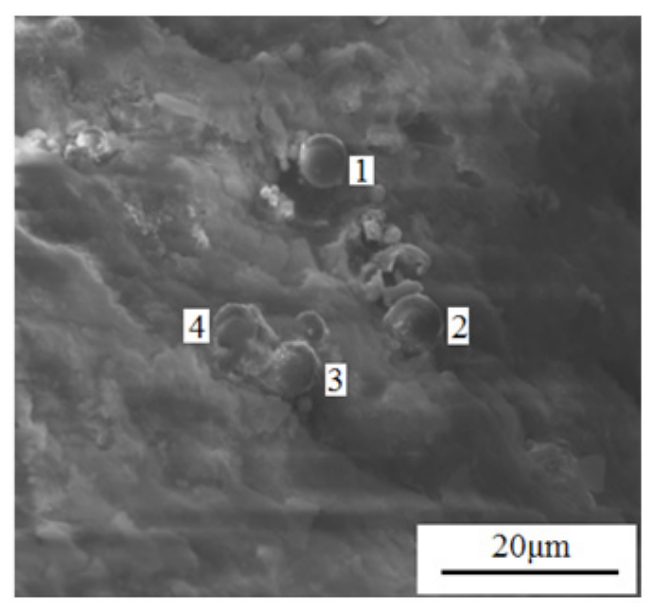

Рис. 1. Вид фрагмента порошка, фиксированного на скотче, при увеличении $4000 \times$, нумерация четырех частиц нанесена авторами.

Fig. 1. The view of a fragment of powder, fixed on scotch tape, with an increase of $4000 \times$, the numbering of the four particles caused by the authors.

основная доля частиц, этому условию соответствует (с точностью до кислорода, ведущего к окислению поверхностного слоя частиц). Однако, имеется и небольшая доля частиц с составом, существенно отличающимся от состава электродов, в частности, с доминированием железа. Подтверждают сказанное карты распределения элементов. В частности, карта распределения железа включает область с относительно крупными четырьмя частицами. На Рис. 2, наряду с уже отмеченными частицами, отрезками белых линий указаны и более мелкие частицы с доминированием железа. Заметим также, что отмеченные частицы явно ферромагнитны.

Разумеется, карты распределения меди и цинка демонстрируют резкое снижение плотности распределения в областях локализации частиц 1-4. Подчеркнем, что карта распределения железа констатирует наличие частиц с диаметрами менее 1 мкм. Скорее всего, и относительно крупные пронумерованные частицы включают шарообразные фрагменты также с диаметрами менее 1 мкм. В Табл. 1 приведен химический состав центральной области частицы, имеющей номер 2 на Рис. 1 и 2. Поскольку диаметр выбранной области того же порядка, что и диаметр частицы 2, химический состав в Табл. 1 отражает некоторое усредненное по составам более мелких частиц содержание элементов.

Прокомментируем данные Табл. 1. Резкое снижение содержания $\mathrm{Cu}$ и $\mathrm{Zn}$ (по сравнению с составом электродов) с одновременным появлением железа,

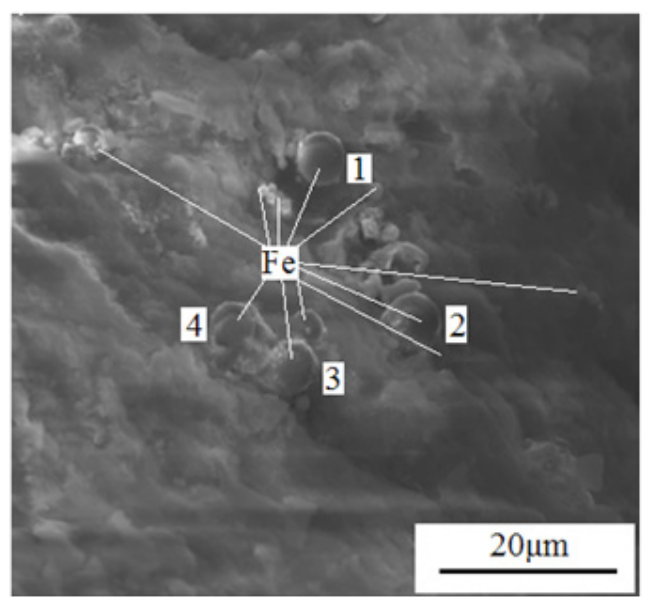

Рис. 2. Частицы с доминированием железа, идентифицированные по картам распределения элементов, включая частицы, отмеченные на Рис. 1.

Fig. 2. Particles with dominance of iron identified by the distribution maps of elements including particles marked in Fig. 1.

доминирующего в составе частицы 2, указывают, на реализацию механизмов трансмутации большей части меди и цинка. Сравнительно высокая доля кислорода указывает, скорее всего, на процессы окисления поверхностного слоя мелких шарообразных частиц. Появление же (в относительно небольших долях) остальных элементов требует отдельного рассмотрения и может быть связано как с процессами синтеза элементов (см. п.3.2), наблюдавшегося в [1] и обсуждавшегося с качественных позиций в [3], [4], так и с процессами, сопутствующими трансмутации меди и цинка. Некоторые искажения в истинный состав частицы 2, по-видимому, вносят спектры от скотча (углерод, сера, кислород, хлор) и предметного столика (алюминий). Кроме того, на содержании хлора должно сказываться его присутствие в исходном растворе.

\section{3. Обсуждение результатов}

\section{1. О механизме трансмутаиии экстрагированных частии, электродов}

В простейшем варианте механизмы трансмутации сводятся К эндотермическим реакциям, которые должны включать испускание легких элементов

$$
\begin{aligned}
& { }_{29} \mathrm{Cu} \rightarrow{ }_{26} \mathrm{Fe}+{ }_{3} \mathrm{Li}, \\
& { }_{30} \mathrm{Zn} \rightarrow{ }_{26} \mathrm{Fe}+{ }_{4} \mathrm{Be} .
\end{aligned}
$$

Напомним [6], медь в природе присутствует в виде двух стабильных изотопов: $\mathrm{Cu}^{63}(69.17 \%), \mathrm{Cu}^{65}(30.83 \%)$,

Табл. 1. Химический состав частицы, отмеченной номером 2 на Рис. 1 и 2.

Table 1. The chemical composition of the particle, marked with number 2 in Figs. 1 and 2.

\begin{tabular}{|c|c|c|c|c|c|c|c|c|c|c|c|c|c|}
\hline Элемент / Element & $\mathrm{C}$ & $\mathrm{O}$ & $\mathrm{Al}$ & $\mathrm{Si}$ & $\mathrm{S}$ & $\mathrm{Cl}$ & $\mathrm{K}$ & $\mathrm{Ca}$ & $\mathrm{Cr}$ & $\mathbf{F e}$ & $\mathrm{Ni}$ & $\mathrm{Cu}$ & $\mathrm{Zn}$ \\
\hline Bec.\% / Wt.\% & 1.07 & 14.05 & 0.28 & 0.69 & 0.43 & 0.56 & 0.36 & 0.30 & 0.30 & $\mathbf{7 2 . 4 1}$ & 0.55 & 5.46 & 3.54 \\
\hline Ат.\% / At.\% & 3.56 & 35.12 & 0.41 & 0.99 & 0.54 & 0.63 & 0.37 & 0.30 & 0.23 & $\mathbf{5 1 . 8 7}$ & 0.37 & 3.43 & 2.17 \\
\hline
\end{tabular}


а цинк в виде четырех: $\mathrm{Zn}^{64}$ (48.6\%), $\mathrm{Zn}^{66}(27.9 \%)$, $\mathrm{Zn}^{67}(4.1 \%), \mathrm{Zn}^{68}(18.8 \%)$, причем имеется и пятый долгоживущий изотоп $\mathrm{Zn}^{70}(0.6 \%)$. Кроме того, при реакциях (1) и (2) следует обсуждать и возможность участия нестабильных изотопов $\mathrm{Li}$ и Ве. Поэтому мы не конкретизировали при записи реакций (1) и (2) массовые числа изотопов. Проведем оценку возможного числа трансмутирующих частиц шаровой формы с радиусом 1 мкм, полагая для простоты, что электроды являются чисто медными, да еще и моноизотопными $\left(\mathrm{Cu}^{63}\right)$, а испускается только изотоп $\mathrm{Li}^{6}$. Тогда реакция

$$
{ }_{29} \mathrm{Cu}^{63}+\Delta \varepsilon \rightarrow{ }_{26} \mathrm{Fe}^{57}+{ }_{3} \mathrm{Li}^{6}
$$

соответствует поглощению энергии $\Delta \varepsilon \approx 19.49$ МэВ.

Частица с радиусом $R$ и атомной плотностью $N_{0}$ содержит

$$
N=N_{0} 4 \pi R^{3} / 3
$$

атомов. Подставляя в (4) значения $R=1 \mathrm{мкм}=10^{-4} \mathrm{~cm}$, $N_{0}=8.52 \cdot 10^{22}\left(\right.$ атом $\left./ \mathrm{cm}^{3}\right)$, получаем $N \approx 3.57 \cdot 10^{11}$. Следовательно, образование такой частицы требует энергии

$$
\Delta E=N \Delta \varepsilon \approx 7 \cdot 10^{12} \mathrm{MэB} \approx 1.1 \text { Дж. }
$$

Энергия $W$ конденсатора емкостью $C$ при разности потенциалов $\Delta \varphi$, как известно, дается формулой $W=C(\Delta \varphi)^{2} / 2$. Значит, в условиях нашего эксперимента $(\Delta \varphi=560 \mathrm{~B}, C=200$ мкФ) разряд конденсатора связан с выделением энергии $W=31.36$ Дж. Так что энергии $W$ хватит для образования не более 30 железных частиц с радиусом 1 мкм. Поэтому неудивительно, что реальные радиусы большинства не полностью трансмутировавших шарообразных частиц оказываются на порядок меньше микрона (10-100 нм). Поскольку поглощаемая энергия пропорциональна $R^{3}$, то при $R=3$ мкм энергии заряженного конденсатора хватило бы не более чем на трансмутацию одной частицы с таким радиусом. Напротив, при $R=0.1$ мкм число трансмутирующих частиц может возрасти на три порядка. Ранее $[7,8]$, нами отмечался факт ограниченности размеров трансмутировавших частиц без оценки энергетического баланса.

Разумеется, для продвижения в понимании природы трансмутации условие соблюдения энергетического баланса желательно дополнить указанием механизма захвата энергии трансмутирующим ядром. Возможный вариант возбуждения ядер, как обсуждалось в [9], связан с поглощением квазипозитрониев (изоэлектронизопозитронных пар), способных переходить из виртуального состояния в состояние с большим временем жизни за счет исходной энергии $W$ заряженного конденсатора. В соответствии с выводами адронной механики [10] о $\pi^{0}$ мезоне как связанном состоянии изоэлектрон-изопозитронной пары можно полагать, что спектр энергий квазипозитрониев принадлежит интервалу (1-135) МэВ. Допустим, что реализуется подобный механизм энергетической накачки ядер. Тогда естественно ожидать, что наибыстрейший вариант их распада связан с испусканием частиц, имеющих нулевой, либо целочисленный (в единицах ћ) спин. Действительно, именно частицы, подчиняющиеся ста- тистике Бозе, способны формировать когерентное состояние Бозе-конденсата. Например, лазерный эффект - генерация когерентных фотонов возникает за счет преобладания индуцированного излучения [11]. Однако для нашего случая более приемлема аналогия со спонтанным когерентным излучением (сверхизлучение Дике [12]). В связи с этим, например, из двух стабильных изотопов $\mathrm{Li}^{6}$ и $\mathrm{Li}^{7}$ в качестве продуктов распада возбужденных ядер изотопов $\mathrm{Cu}^{63}$ и $\mathrm{Cu}^{65}$ предпочтение следует отдать $\mathrm{Li}^{6}$ (спин 1), а не $\mathrm{Li}^{7}$ (спин 3/2). Соответственно, и образовавшееся распределение изотопов железа будет существенно отличаться от природного в пользу нарастания доли изотопа $\mathrm{Fe}^{57}$ (за счет распадов $\mathrm{Cu}^{63}$ ), хотя и изотоп $\mathrm{Fe}^{56}$ должен присутствовать (за счет распадов $\mathrm{Zn}^{64}$ с испусканием $\left.\mathrm{Be}^{8}\right)$. Полезно иметь в виду, что в природном распределении преобладает $\mathrm{Fe}^{56}(\approx 91.75 \%)$, а на изотоп $\mathrm{Fe}^{57}$ приходится ( $\left.\approx 2.12 \%\right)$. В любом случае маловероятно появление изотопа $\mathrm{Fe}^{54}$, доля которого в природном распределении ( $\approx 5.84 \%)$ уступает только $\mathrm{Fe}^{56}$.

Уместно подчеркнуть, что в [1] среди возникших при плазменном электролизе воды химических элементов отмечается присутствие в небольшом количестве $\mathrm{Li}$ и Be. Поскольку $\mathrm{Be}^{8}$ нестабилен (распадается на две a-частицы) речь может идти только о единственном стабильном изотопе $\mathrm{Be}^{9}$. Конечно, появление $\mathrm{Be}^{9}$, скорее всего, связано с процессом синтеза - реакцией поглощения протона при взаимодействии $\mathrm{Be}^{8}$ с квазинейтроном [9]. Полезно напомнить, что понятие квазинейтрона охватывает широкий набор состояний от нейтрона по Сантилли $[10,13,14]$ до гидрино Миллса $[15,16]$. Однако, не исключено, что, несмотря на полуцелый спин, $\mathrm{Be}^{9}$ участвует в распаде возбужденных $\mathrm{Zn}^{66}$ и $\mathrm{Zn}^{67}$, приводя к вкладу в содержание изотопов $\mathrm{Fe}^{57}$ и $\mathrm{Fe}^{58}$. По тем же причинам в качестве конечного продукта может появляться и изотоп $\mathrm{Li}^{7}$, приводя при распаде возбужденных ядер $\mathrm{Cu}^{63}$ и $\mathrm{Cu}^{65}$ к дополнительным вкладам в содержание изотопов $\mathrm{Fe}^{56}$ и $\mathrm{Fe}^{58}$. Фиксация Li и Be в [1] косвенно свидетельствует в пользу предложенной трактовки трансмутации части экстрагированных шарообразных фрагментов латуни. Поскольку в используемой методике микроанализа легкие элементы не идентифицируются, планируется дополнить наблюдения регистрацией оптического спектра возбужденных атомов в ходе электрических разрядов, а также использовать и неспектроскопические варианты анализа. Особую же ценность имеет изотопный анализ для железа и лития.

\section{2. О возможных реакииях синтеза элементов}

Наблюдаются и частицы менее симметричной формы, химические составы которых значительно отличаются друг от друга, как и от состава, приведенного в Табл. 1. Один из вариантов состава, заметно обогащенного $\mathrm{Mg}$ и $\mathrm{Si}$, представлен в Табл. 2.

Прокомментируем данные Табл. 2.

Элементный состав близок (за исключением $\mathrm{Mg}$ ) к элементному составу в Табл. 1, но доли элементов существенно отличаются. Возникновение $\mathrm{Mg}$ и $\mathrm{Si}$ может 
Табл. 2. Химический состав частицы, обогащенный $\mathrm{Mg}$ и $\mathrm{Si}$.

Table 2. The chemical composition of the particles enriched in $\mathrm{Mg}$ and $\mathrm{Si}$.

\begin{tabular}{|c|c|c|c|c|c|c|c|c|c|c|c|c|c|c|}
\hline Элемент / Element & $\mathrm{C}$ & $\mathrm{O}$ & $\mathrm{Mg}$ & $\mathrm{Al}$ & $\mathrm{Si}$ & $S$ & $\mathrm{Cl}$ & $\mathrm{K}$ & $\mathrm{Ca}$ & $\mathrm{Cr}$ & $\mathrm{Fe}$ & $\mathrm{Ni}$ & $\mathrm{Cu}$ & $\mathrm{Zn}$ \\
\hline Bec. $\%$ / Wt. $\%$ & 4.73 & 30.79 & 8.97 & 0.60 & 13.71 & 1.04 & 3.04 & 2.57 & 0.59 & 1.08 & 5.84 & 0.70 & 16.94 & 9.39 \\
\hline Ат.\% / At.\% & 9.98 & 48.80 & 9.35 & 0.57 & 12.38 & 0.83 & 2.17 & 1.67 & 0.38 & 0.53 & 2.65 & 0.30 & 6.76 & 3.64 \\
\hline
\end{tabular}

происходить по нескольким сценариям, в частности, в процессе распада железа. Однако этот распад является эндотермическим процессом, и явно предпочтительнее экзотермические реакции синтеза. Например, при парных столкновениях имеем:

$$
\begin{array}{ll}
{ }_{8} \mathrm{O}^{16}+{ }_{8} \mathrm{O}^{16} \rightarrow{ }_{14} \mathrm{Si}^{28}+\alpha, & \Delta \varepsilon \approx+9.594 \mathrm{M \ni B}, \\
{ }_{6} \mathrm{C}^{12}+{ }_{8} \mathrm{O}^{16} \rightarrow{ }_{14} \mathrm{Si}^{28}, & \Delta \varepsilon \approx+16.756 \mathrm{M \ni B}, \\
{ }_{6} \mathrm{C}^{12}+{ }_{8} \mathrm{O}^{16} \rightarrow{ }_{12} \mathrm{Mg}^{24}+\alpha, & \Delta \varepsilon \approx+6.772 \mathrm{M \ni B}, \\
{ }_{6} \mathrm{C}^{12}+{ }_{6} \mathrm{C}^{12} \rightarrow{ }_{12} \mathrm{Mg}^{24}, & \Delta \varepsilon \approx+13.934 \mathrm{M \ni B} .
\end{array}
$$

Заметим, что синтез $\mathrm{Mg}$ возможен и в отсутствии углерода в ходе экзотермических реакций, учитывающих взаимодействия не только с одним, но с двумя или даже с тремя и четырьмя ядрами, как ионов кислорода, так и продуктов реакции, в силу существования молекулярных кластеров воды - типичны тетраэдрические кластеры $[17,18]$. Ясно, что подобные реакции возможны вблизи границы между жидкостью и паром. Рассматривая также реакции с участием квазинейтронов [9], захвата и испускания электронов и учитывая, что расширяется изотопный состав кислорода, углерода, магния и кремния, можно получить (существенно расширив список реакций (5-8)) весь состав элементов (с набором стабильных изотопов), перечисленных в Табл. 2.

Отметим также, что форма частицы гораздо ближе к пластинчатой, чем к равноосной. Размеры ее «уплощенной» поверхности $\approx(4 \times 5)$ мкм заметно превышают толщину. Частица лежит на более массивной пластинчатой подложке экстрагированного материала с составом, близким к составу электродов. Значит, содержание меди и цинка, приведенные в Табл. 2, могут в основном отражать состав подложки. В пользу этого свидетельствует близость отношения $6.76 / 3.64 \approx 1.86$ долей меди и цинка (см. Табл. 2) с отношением их долей в составе электродов $63.77 / 36.23 \approx 1.76$. Следовательно, в составе частицы реальное содержание меди и цинка, связанное с их генерацией из воды, может быть существенно меньше, чем в Табл. 2. Таким образом, имеются аргументы в пользу выводов [2] о реализации синтеза элементов при плазменном электролизе воды с помощью простой демонстрационной методики. Кроме того, размер «уплощенной» поверхности частицы соответствует, по-видимому, характерному для условий эксперимента масштабу области взаимодействия ионов кислорода, квазинейтронов и продуктов реакций, приведшему к формированию обсуждаемой частицы твердого осадка.

Существенную роль в эрозии материала электродов, по-видимому, играют энергетические всплески на их по- верхностях, связанные с кавитационными коллапсами пузырьков пара. Ясно, например, что нелинейные волны, порождаемые кавитационным эффектом, должны способствовать отрыву фрагментов поверхностных слоев электродов, в первую очередь, из зон локального плавления. Заметим, что с кавитационным эффектом связывают и сценарии синтеза элементов $[19,20]$.

\section{4. Заключение}

Микроанализ твердого осадка, полученного в ходе электролиза поваренной соли в воде (как и дистиллированной воды) показал, что использование упрощенной, по сравнению с [1], методики плазменного электролиза подтверждает, в принципе, выводы в [1] и [2] о синтезе химических элементов из воды.

Однако имеется и существенное уточнение. А именно, есть основания считать, что доля некоторых элементов, присутствующих в твердом осадке, связана не только с синтезом элементов, но и с трансмутацией элементов, входящих в состав электродов, испытывающих эрозию в ходе электролиза. В частности, это относится к железу. Достаточно характерны шарообразные частицы с повышенной концентрацией железа, имеющие радиусы $R$ из диапазона $(10-100)$ нм.

Процесс трансмутации меди и цинка, ведущий к образованию железа, требует поглощения энергии. Это позволяет выполнить оценку количества трансмутировавших частиц с радиусом $R$ на основе баланса между выделяемой при разряде и поглощаемой в процессе трансмутации энергии. Оценка сразу проясняет величину критического размера для таких частиц. Механизм захвата энергии ядрами предположительно связывается с поглощением квазипозитрониев. Для железа, полученного при трансмутации латунных электродов, возможно существенное отклонение от природного изотопного состава.

Благодарности/Acknowledgements. Авторь выражают благодарность В.А. Панькову и Б.П. Кузьмину за инициацию постановки экспериментальных исследований, подтверждающих синтез элементов. Авторы признательны участникам тринадияатого международного уральского семинара «Радиационная физика металлов и сплавов» за обсуждение части результатов работы. / The authors are grateful to V.A. Pan'kov and B.P. Kuzmin for initiating the production of experimental studies confirming the synthesis of elements. The authors are grateful to the participants of the thirteenth international Ural seminar "Radiation Physics of Metals and Alloys" for discussing some of the results of the work. 


\section{Литература/References}

1. V.F. Balakirev, V.V. Krymskiy, B.V. Bolotov et al. Vzaimoprevrashcheniya khimicheskikh elementov. Yekaterinburg, UB RAS (2003) 97 p. (in Russian) [В.Ф. Балакирев, В. Крымский, Б.В. Болотов и др. Взаимопревращения химических элементов. Екатеринбург, УрО РАН (2003) 97 с.]

2. V.A. Pan'kov, B.P. Kuzmin. Aktual'nyye problemy sovremennoy nauki. 5, 117 (2008). (in Russian) [B. А. Паньков, Б. П. Кузьмин. Актуальные проблемы современной науки. 5, 117 (2008).]

3. M.P. Kashchenko, V.F. Balakirev. Letters on materials. 8 (2), 152 (2018). (in Russian) [М. П. Кащенко, В. Ф. Балакирев. Письма о материалах. 8 (2), 152 (2018).] Crossref

4. M.P. Kashchenko, V.F. Balakirev. Letters on materials. 7 (4), 380 (2017). Crossref

5. M.M. Krishtal, I.S. Yasnikov et al. Mir fiziki i tekhniki. Skaniruyushchaya elektronnaya mikroskopiya i rentgenospektral'nyy mikroanaliz v primerakh prakticheskogo primeneniya.Moscow, Tekhnosfera(2009) 208 p. (in Russian) [М. М. Криштал, И. С. Ясников и др. Мир физики и техники. Сканирующая электронная микроскопия и рентгеноспектральный микроанализ в примерах практического применения. Москва, Texносфера (2009) 208 c.]

6. Table of Nuclides. Website

7. M.P. Kashchenko, V.F. Balakirev, M.B. Smirnov, Yu. L. Chepelev, V. V. Ilushin, N. V. Nikolaeva, V. G. Pushin. The thirteenth international Ural seminar "Radiation damage physics of metals and alloys". (February 24March 2, Kyshtym, Russia) Abstracts. Yekaterinburg, IPM UB RAS (2019) p. 68. (in Russian) [М.П. Кащенко, В.Ф. Балакирев, М.Б. Смирнов, Ю.Л. Чепелев, В.В. Илюшин, Н.В. Николаева, В.Г. Пушин. Тринадцатый международный уральский семинар «Радиационная физика металлов и сплавов». (24 февраля - 2 марта, 2019, Кыштым, Россия). Тезисы докладов. Екатеринбург, ИФМ УрО РАН (2019) с. 68.]
8. M.P. Kashchenko, V.F. Balakirev, M.B. Smirnov, Yu.L. Chepelev, V.V. Ilushin, N.V. Nikolaeva, V.G. Pushin. International conference "Materials science of the future: research, development, scientific training" (February 12-14, 2019, Nizhni Novgorod, Lobachevsky University): Abstracts. Nizhni Novgorod, Nizhni Novgorod University Press (2019) p. 40.

9. M.P. Kashchenko, N.M. Kashchenko. Letters on materials. 9 (3), 316 (2019). (in Russian) [М.П. Кащенко, H.M. Кащенко. Письма о материалах. 9 (3), 316 (2019).] Crossref

10. R. M. Santilli. Foundations of Hadronic Chemistry. With Applications to New Clean Energies and Fuels. London, Kluwer Academic Publishers (2001) 554 p.

11. H. Haken. Zs. Phys. 181, 96 (1964). Crossref

12. R. H. Dicke. Phys. Rev. 93, 99 (1954). $\underline{\text { Crossref }}$

13. R. Norman, A. A. Bhalekar, S. Beghella, B. B. Buckley, J. Dunning-Davies, J. Rak, R.M. Santilli. American Journal of Modern Physics. 6 (4-1), 85 (2017). Crossref

14. R. M. Santilli. International Journal of Applied Physics and Mathematics. 9 (2), 72 (2019). Crossref

15. R. L. Mills. The Grand Unified Theory of Classical Physics. BlackLight Power, Cranbury, New Jersey (2011).

16. R. Mills, Y. Lu, R. Frazer. Chinese Journal of Physics. 56, 1667 (2018). $\underline{\text { Crossref }}$

17. T. Tokushima, Y. Harada, O. Takahashi, Y. Senba, H. Ohashi, L. G. M. Pettersson, A. Nilsson, S. Shin. Chem. Phys. Lett. 460, 387 (2008). Crossref

18. C. Huang, K. T. Wikfeldt, T. Tokushima, D. Nordlund, Y. Harada, U. Bergmann, M. Niebuhr, T.M. Weiss, Y. Horikawa, M. Leetmaa, M. P. Ljungberg, O. Takahashi, A. Lenz, L. Ojamae, A.P. Lyubartsev, S. Shin, L. G. M. Pettersson, A. Nilsson. PNAS. 106, 15214 (2009). Crossref

19. C. G. Camara, S. D. Hopkins, K. S. Suslick, S. J. Putterman. Phys. Rev. Lett. 98 (6), 064301 (2007). Crossref

20. R. I. Nigmatulin, R. T. Lahey, R. P. Taleyarkhan, C. D. West, R. C. Block. Phys. Usp. 57, 877 (2014) . Crossref 